\title{
Expression Pattern of Receptor Activator of NFik (RANK) in a Series of Primary Solid Tumors and Related Bone Metastases
}

DANIELE SANTINI,' GIUSEPPE PERRONE, ${ }^{2}$ ILARIA ROATO, ${ }^{3}$ LAURA GODIO, ${ }^{4}$ FRANCESCO PANTANO,' DONATELLA GRASSO, ${ }^{5}$ ANTONIO RUSSO, ${ }^{6 *}$ BRUNO VINCENZI,'

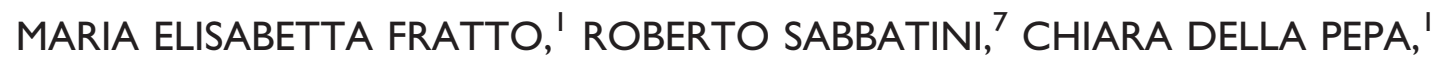
CAMILLO PORTA, ${ }^{5}$ ALESSANDRO DEL CONTE, ${ }^{8}$ GAIA SCHIAVON, ${ }^{\prime}$ ALFREDO BERRUTI, ${ }^{9}$ ROSA MARIA TOMASINO, ${ }^{10}$ MAURO PAPOTTI, ${ }^{4}$ NICOLA PAPAPIETRO, ${ }^{\prime \prime}$ ANDREA ONETTI MUDA, ${ }^{2}$ VINCENZO DENARO, " AND GIUSEPPE TONINI'

'Department of Medical Oncology, University Campus Bio-Medico, Rome, Italy

${ }^{2}$ Department of Surgical Pathology, University Campus Bio-Medico, Rome, Italy

${ }^{3}$ Cell Therapeutics, Inc., Bresso, Milan, Italy

${ }^{4}$ Department of Pathology, University of Turin, Torino, Italy

${ }^{5}$ Department of Medical Oncology, Università and Istituto di Ricovero e Cura a Carattere Scientifico Policlinico S. Matteo, Pavia, Italy

${ }^{6}$ Department of Surgical and Oncological Sciences, Section of Medical Oncology, University of Palermo, Palermo, Italy

${ }^{7}$ Department of Medical Oncology, Università degli Studi di Modena e Reggio Emilia, Modena, Italy

${ }^{8}$ Department of Medical Oncology, Ospedale di Empoli, Empoli, Italy

${ }^{9}$ Department of Medical Oncology, Azienda Ospedaliera Universitaria San Luigi di Orbassano, Torino, Italy

${ }^{10}$ Department of Pathology, Università degli Studi di Palermo, Palermo, Italy

"'Department of Orthopedics, University Campus Bio-Medico, Rome, Italy

Receptor activator of NFKB ligand (RANKL), RANK, and osteoprotegerin (OPG) represent the key regulators of bone metabolism both in normal and pathological conditions, including bone metastases. To our knowledge, no previous studies investigated and compared RANK expression in primary tumors and in bone metastases from the same patient. We retrospectively examined RANK expression by immunohistochemistry in 74 bone metastases tissues from solid tumors, mostly breast, colorectal, renal, lung, and prostate cancer. For 40 cases, tissue from the corresponding primary tumor was also analyzed. Sixty-six (89\%) of the 74 bone metastases were RANK-positive and, among these, 40 (59.5\%) showed more than $50 \%$ of positive tumor cells. The median percentage of RANK-positive cells was $60 \%$ in primary tumors and metastases, without any statistically significant difference between the two groups $(P=0.194)$. The same percentage was obtained by considering only cases with availability of samples both from primary and metastasis. Our study shows that RANK is expressed by solid tumors, with high concordance between bone metastasis and corresponding primary tumor. These data highlight the central role of RANK/RANKL/OPG pathway as potential therapeutic target not only in bone metastasis management, but also in the adjuvant setting. J. Cell. Physiol.

J. Cell. Physiol. 226: 780-784, 20II. (C) 2010 Wiley-Liss, Inc.

Receptor activator of NFKB ligand (RANKL)/RANK/ osteoprotegerin (OPG) pathway represents a key regulator of bone metabolism both in normal and pathological conditions, including bone metastases. RANK is expressed at the surface of osteoclasts and it is an essential signaling receptor in osteoclast differentiation. RANKL is preferentially expressed on committed pre-osteoblastic cells and, upon binding to RANK, has been shown to both activate mature osteoclasts and mediate osteoclastogenesis in presence of macrophage-colony stimulating factor (M-CSF). OPG, produced by osteoblast lineage cells, acts as a decoy receptor and inhibits osteoclast formation, function, and survival by preventing RANKL binding to RANK (Boyce and Xing, 2008). RANK expression has been found in osteosarcoma cell lines from human origin together with some human osteosarcoma specimens, both at transcriptional and protein level (Wittrant et al., 2006; Mori et al., 2007a).

However, RANK expression is not restricted to bone cells and has been also observed in other tissues including epithelial

*Correspondence to: Antonio Russo, Section of Medical Oncology,

Department of Surgical and Oncological Sciences, Università di

Palermo, Via del Vespro 127, 90127 Palermo, Italy.

E-mail: lab-oncobiologia@usa.net

Received 28 March 2010; Accepted 13 August 2010

Published online in Wiley Online Library

(wileyonlinelibrary.com), 20 September 2010.

DOI: 10.1002/jcp.22402 
cells of mammary gland, lung, brain, and kidney (Nakagawa et al., 1998; Hsu et al., 1999; Srivastava et al., 2003). Moreover, RANK is also expressed in several cancer cell lines where seems to play a central role in tumor cell migration and invasion (Mori et al., 2007b). As recently demonstrated, this pathway has been found to be disregulated in several solid tumors, such as breast cancer, malignant bone tumors, multiple myeloma, giant cell tumors of bone, chondroblastoma, neuroblastoma, squamous cell carcinoma, and Hodgkin disease (Mori et al., 2007b).

Additionally, Jones et al. (2006) have reported the expression of functional RANK in human breast and prostate cancer and mouse melanoma cell lines. Moreover, Chen et al. studied RANKL, RANK, and OPG expression in primary and metastatic prostate cancer samples. They found that the expression of these molecules was significantly higher in metastatic prostate cancer than in primary carcinoma (Chen et al., 2006). Similarly, Brown et al. (2004) analyzed primary and metastatic prostate cancer samples and showed that the proportion of tumor cells expressing RANKL was significantly increased in bone metastases compared with metastases in other sites or with the primary tumor. Furthermore, Mori et al. evaluated cases of primary $\mathrm{HCC}$ and observed that RANKL expression in HCC cells is correlated with the development of bone metastases after hepatic resection (Sasaki et al., 2007). Moreover, it has been demonstrated that RANKL triggers cytoskeletal changes and migration of several human epithelial tumor cells (including primary breast cancer cells) expressing RANK (Jones et al., 2006).

Taken together, these findings suggest that, upon RANKL activation, RANK may stimulate primary tumor cells to migrate into the bone and may induce the bone metastases process, with additional autocrine and paracrine mechanisms.

To our knowledge, there are no previous studies investigating RANK expression in primary tumors and corresponding bone metastases. Thus, we studied RANK expression by immunohistochemistry (IHC) in a large heterogeneous cohort of human primary solid tumors and related bone metastases, in order to compare RANK expression between the two sites of tumoral disease. These results drive us to investigate if RANK expression depends more on bone microenvironment or on primary tumor histology and whether these molecules could represent ubiquitariously expressed targets for anticancer therapies.

\section{Materials and Methods Patients}

Surgical biopsy samples from 74 patients with bone metastases were examined in this study. The period of accrual was March 2006-January 2009. All patients had histological diagnosis of solid tumor (regardless of treatment performed) and signed the informed consent. For 40 patients, the paraffined samples of both primary and metastatic tumors were available. For 34 patients we only examined the samples from bone metastasis. Exclusion criteria were any previous hematological disease (myelodisplastic, lymphoproliferative, and myeloproliferative syndromes) and assumption of drugs with high osteotrophism, such as bisphosphonates, calcitonin, and Vitamin D. Table I shows the distribution of patients included in the study, by site of primary tumor.

\section{Immunohistochemistry}

IHC experiments were performed on surgical pathology specimens used for diagnosis. Samples were fixed in $4 \%$ neutral buffered formaldehyde and, in case of bone biopsies, were demineralized by EDTA solution ( $1,000 \mathrm{ml}$ water, $37,22 \mathrm{~g}$ ethylenediaminetetraacetic acid disodium salt dehydrate, $70 \mathrm{ml}$ $\mathrm{HCL} 37 \%$ ) for less than $3 \mathrm{~h}$. Bones specimens were washed in water before paraffin embedding.

Representative tumor blocks were sectioned at $3 \mu \mathrm{m}$ thickness. $\mathrm{IHC}$ was performed by the streptavidin-biotin method.

Endogenous peroxidase in the section was blocked by incubation with 3\% hydrogen peroxide. A mouse monoclonal antibody against RANK protein (clone 80707, R\&D Systems, Inc., Minneapolis, MN) was used as primary antibody at a concentration of $25 \mu \mathrm{g} / \mathrm{ml}$. Sections were incubated with LSAB2 (Dakocytomation, Carpinteria, CA). 3-3'-Diaminobenzidine (DAB) was used for color development and hematoxylin was used for counterstaining. Negative controls were obtained by omitting primary antibodies.

Scoring for RANK was based on the relative staining intensity of tumor cells compared to the RANK staining of osteoclasts (bone metastatic lesions) and tissue associated macrophages (primary tumors). These internal references were then used as internal positive controls between slides and samples as well as for the staining procedure. Each pathologic tissue was evaluated by comparison with the internal controls. Staining intensity was graded as absent $(0)$, positive but less intense than internal control tissue $(I+)$, positive like internal control tissue $(2+)$, positive but more intense than internal control tissue $(3+)$. Samples with

TABLE I. Number of samples for each type of primary tumor and metastases

\begin{tabular}{|c|c|c|c|c|}
\hline & \multicolumn{2}{|c|}{ Primary } & \multicolumn{2}{|c|}{ Metastatic } \\
\hline & $\begin{array}{l}\text { No. of } \\
\text { lesions }\end{array}$ & $\begin{array}{l}\text { No. (\%) of lesions } \\
\text { RANK positive in }>50 \% \\
\text { of tumor tissue }\end{array}$ & $\begin{array}{l}\text { No. of } \\
\text { lesions }\end{array}$ & $\begin{array}{l}\text { No. (\%) of lesions } \\
\text { RANK positive in }>50 \% \\
\text { of tumor tissue }\end{array}$ \\
\hline Breast & 14 & 8 (57.1\%) & 19 & 13 (68.4\%) \\
\hline Colorectal & 6 & $4(66.6 \%)$ & 8 & $5(62.5 \%)$ \\
\hline Renal & 5 & $2(40 \%)$ & 6 & $2(33.3 \%)$ \\
\hline Lung & I & I (100\%) & 8 & 7 (87.5\%) \\
\hline Prostate & 4 & $2(50 \%)$ & 7 & $2(28.5 \%)$ \\
\hline Thymic & i & $0(0 \%)$ & I & $0(0 \%)$ \\
\hline Esophageal & I & I (100\%) & I & $\mathrm{I}(100 \%)$ \\
\hline Bladder & I & $0(0 \%)$ & 2 & I $(50 \%)$ \\
\hline Thyroid & 2 & $0(0 \%)$ & 5 & $0(0 \%)$ \\
\hline Hepatic & I & $\mathrm{I}(100 \%)$ & 2 & I $(50 \%)$ \\
\hline Cervical & 2 & $2(100 \%)$ & 2 & $2(100 \%)$ \\
\hline Endometrial & 2 & I (50\%) & 2 & $2(100 \%)$ \\
\hline Biliary tract & & & I & $0(0 \%)$ \\
\hline Salivary glands & & & 2 & I (50\%) \\
\hline Squamous & & & 1 & I (100\%) \\
\hline Malignant melanoma & & & I & I (100\%) \\
\hline Unknown & & & 6 & I (16.6\%) \\
\hline
\end{tabular}


regions of heterogeneous staining intensities of RANK were scored and the percentage of each area was recorded. Tumor cells with an immunostaining intensity of $2+$ and $3+$ were considered as positive. Immunostaining was assessed by two independent pathologists blinded to clinical characteristics and outcomes.

\section{Statistical analysis}

Wilcoxon signed-rank test was used to assess differences in RANK expression between primary and related metastatic lesions. $P=0.05$ was considered as statistically significant. SPSS software (version 14.00; SPSS, Inc., Chicago, IL) was used for statistical analysis.

\section{Results}

\section{RANK protein expression}

RANK immunostaining, if present, was observed both at the plasma membrane and the cytoplasm of tumor cells (Fig. I). Consistently with findings from other groups, RANK staining was also constantly found in osteoclast cells of bone tissue around metastatic lesions (Nakagawa et al., 1998) and in tissue associate macrophages within and around primary cancers (van Ravenswaay Claasen et al., 1992; Lau et al., 2007). Thus, both cell types were used as internal positive controls.

\section{RANK expression in bone metastases}

Sixty-six (89\%) of the 74 bone metastasis samples were RANKpositive and, among these, 40 (59.5\%) showed more than $50 \%$ of positive tumor cells.

The median percentage of RANK-positive cells seemed to be dependent on the tumor histotype, being $70 \%$ for breast, $60 \%$ for colorectal, $30 \%$ for renal, $100 \%$ for lung, and $40 \%$ for prostate cancer. We did not perform analysis of differences because of the heterogeneous distribution of the cases.

To note, the only case of bone metastasis from thymoma was RANK-negative, whereas the median percentage of RANKpositive cells was between $30 \%$ for tumor of unknown origin and $100 \%$ for metastases from esophageal cancer. Specifically, the median RANK expression was $40 \%$ in bladder cancer, thyroid cancer and cholangiocarcinoma, $55 \%$ in salivary glands tumors, $60 \%$ in hepatocarcinoma, $70 \%$ in cervical cancer, $75 \%$ in endometrial cancer, $80 \%$ in squamous cancer, and $90 \%$ in melanoma (Fig. 2).

\section{RANK expression in primary tumors}

Considering only the 40 primary tumors samples, 27 (67.5\%) were RANK-positive and 22 (55\%) showed more than $50 \%$ of positive tumor cells.

Also at primary sites, the median percentage of RANKpositive cells seemed to be dependent on the tumor histotype, being $65 \%$ for breast, $75 \%$ for colorectal, $25 \%$ for renal, $90 \%$ for lung, and $55 \%$ for prostate cancer. The number of primary lesions did not allow a reliable difference analysis evaluation. Considering all the histotypes, we observed that RANK expression was considerably variable among tumors. For example, thymoma and bladder cancers were completely negative, whereas the median percentage of RANK-positive cells was between $15 \%$ and $85 \%$ in the other tumors (thyroid cancer and cervical cancer, respectively). Specifically, RANK expression was $30 \%$ in endometrial cancer, $60 \%$ in hepatocarcinoma and $80 \%$ in esophageal cancer (Fig. 3).

\section{Comparison of RANK expression between primary tumors and bone metastases}

To compare the expression of RANK in primary tumor and metastasis, we calculated the median percentage of RANKpositive cells in all the samples from the primaries versus metastases. The median value of RANK-positive cells was $60 \%$
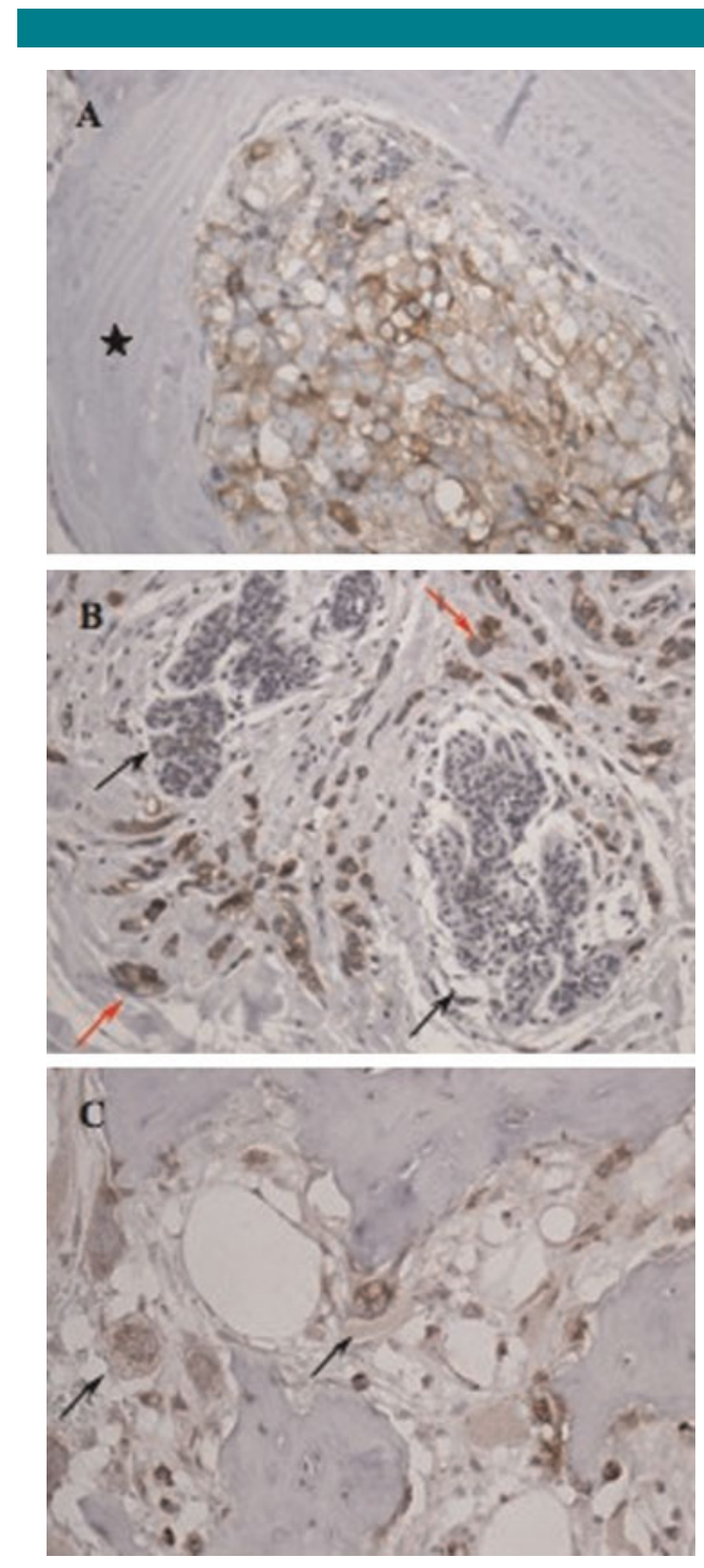

Fig. I. Immunohistochemical results. Image A shows a prostatic bone metastasis in which the majority of tumor cells are positive for RANK immunostaining. The black star is placed on the peripheral bone tissue. In image B, one of the breast cancer studied. The tumor cells are strongly positive for RANK immunostaining (red arrows). The normal glandular epithelium is negative or weakly positive (black arrows) confirming the RANK protein overexpression of the tumor tissue. Image C shows osteoclast cells positive for RANK staining (black arrows). This picture is taken from a perimetastatic area. Original magnification A,C $400 \times$, B $100 \times$. [Color figure can be viewed in the online issue, which is available at wileyonlinelibrary.com.]

in both groups. In terms of percentage of RANK-positive cells, we did not find any statistically significant difference between primary and metastases group $(P=0.194)$ (Fig. 4). Moreover, we repeated the analysis including only patients with availability of matched primary and metastasis tissue samples and we 


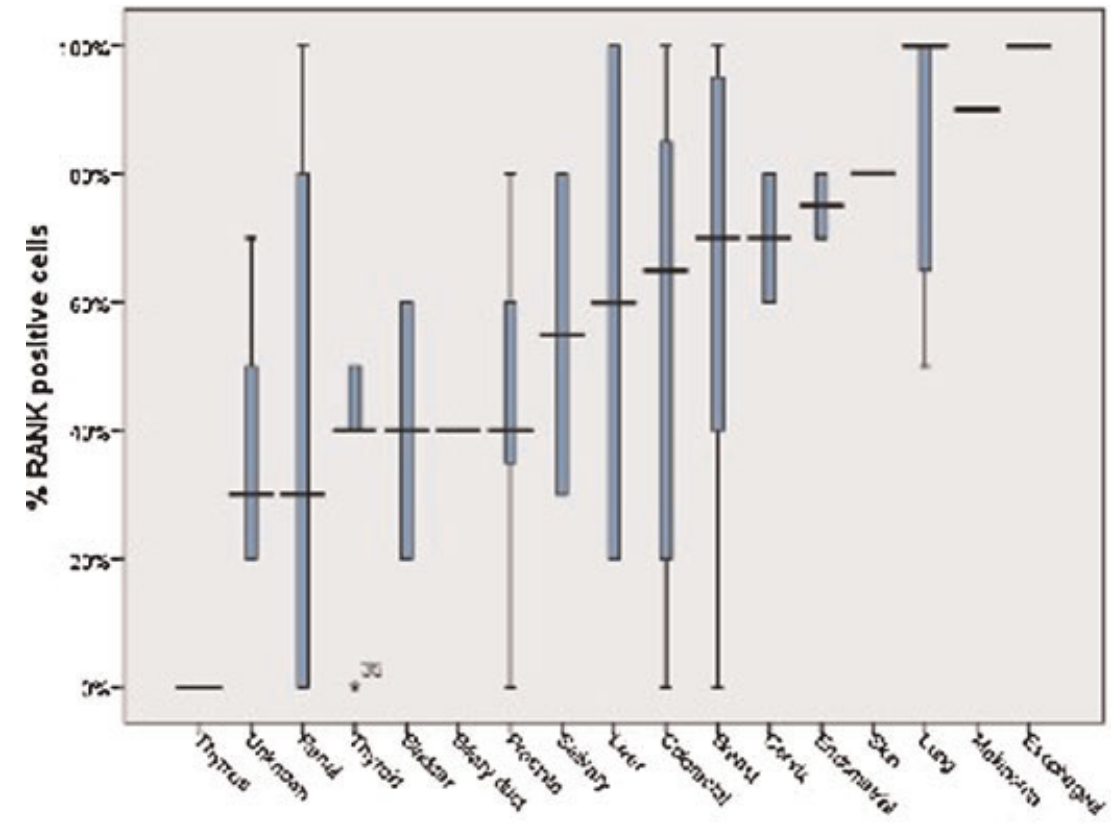

Fig. 2. shows the distribution of RANK-positive cells among the bone metastases.

observed a median percentage of RANK-positive cells of $60 \%$ for the "primary" and 55\% for the "metastasis" group $(P=0.528)$ (Fig. 5). Moreover, considering only patients with breast cancer ( 14 primaries and 19 bone metastases), we did not observe any statistically significant difference $(P=0.854)$ between number of RANK-positive cells in primary $(65 \%)$ versus bone metastasis (70\%). No statistical differences were found between primary and metastatic prostate and renal cancers $(P=0.775$ and 0.925 , respectively).

\section{Conclusions}

RANK/RANKL/OPG pathway represents a new therapeutic target in the treatment of osteoporosis and in the risk reduction of skeletal related events in bone metastatic cancer patients (Cummings et al., 2009; Fizazi et al., 2009; Smith et al., 2009). Our study shows that RANK is expressed in a wide percentage of bone metastases deriving from several different primary histotypes and in a large part of corresponding primary solid

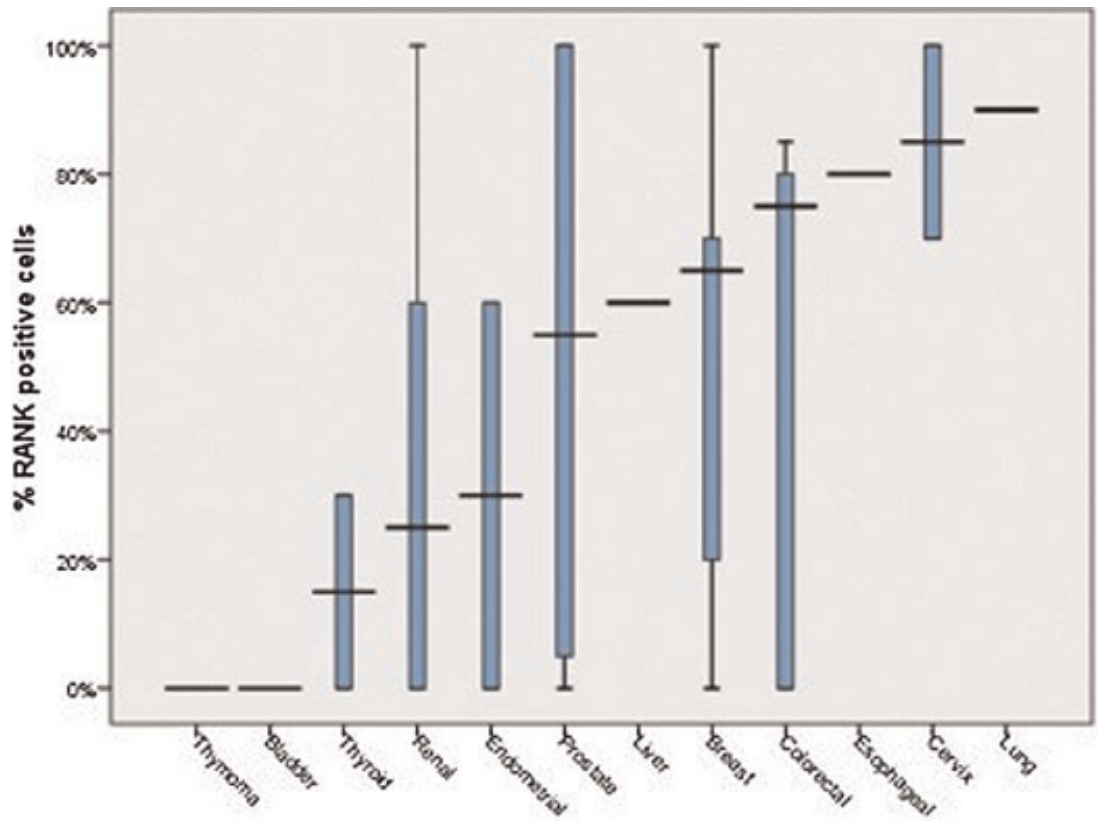

Fig. 3. Distribution of RANK-positive cells among the primitive tumors. 


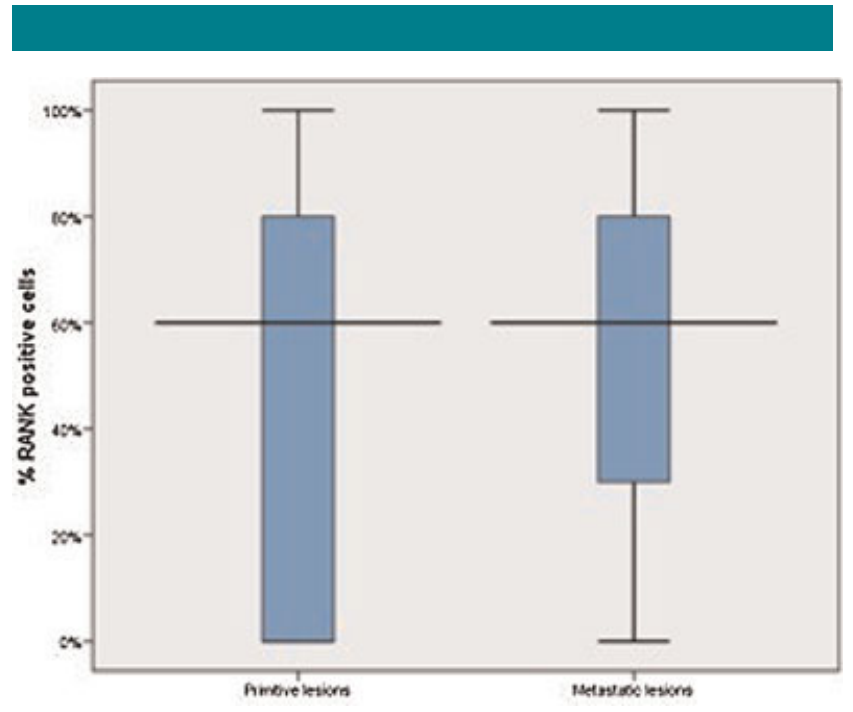

Fig. 4. Comparison of the RANK expression between "primary" and "metastasis" considering all the samples. [Color figure can be viewed in the online issue, which is available at wileyonlinelibrary.com.]

tumors. Further studies are obviously necessary to confirm these data and to verify the functional importance of RANK expression in each histotype. However, these results open a new scenario in which RANK/RANKL/OPG pathway could become an optimal therapeutic target for anticancer therapy. Moreover, we observed that RANK expression in primary tumors and in the related bone metastases differs according to the histotype and not according to the metastatization process or to the bone biological microenvironment. There are tumors expressing high levels of RANK both in primary and bone metastasis (e.g., breast and colorectal cancers) compared to others expressing low levels in both sites (e.g., prostate cancer). Unfortunately, the heterogeneous distribution of primaries did not allow a statistical analysis to compare the different levels of RANK expression according to the histological origin of tumors.

However, this study clearly shows for the first time in literature that, considering all primary sites, RANK expression

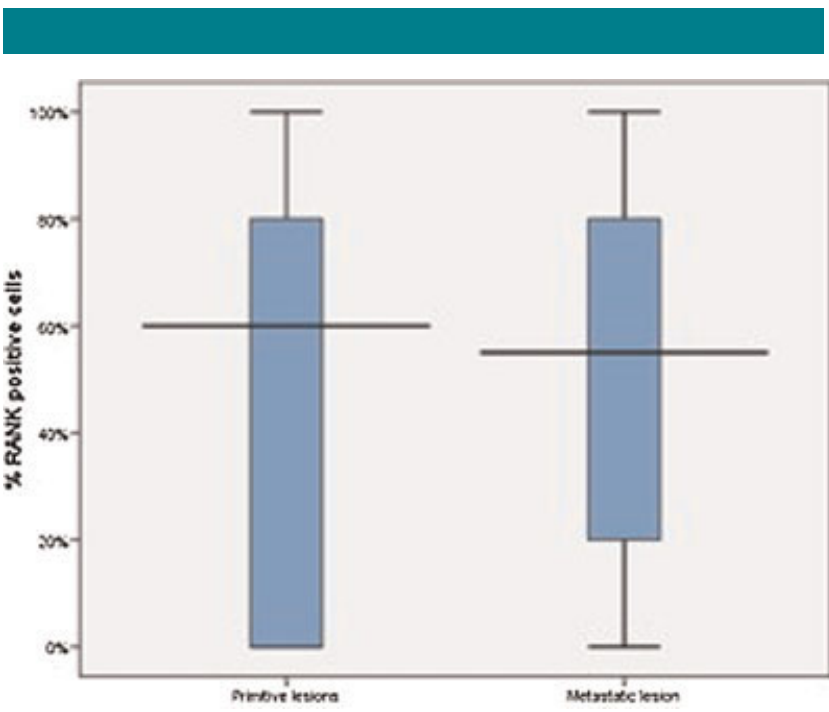

Fig. 5. Comparison of the RANK expression between "primary" and "metastasis" considering only the samples with both the "primary' and the "metastasis" available. [Color figure can be viewed in the online issue, which is available at wileyonlinelibrary.com.] level in bone metastases is, on average, not significantly different from the level detected in the related primary tumors. For this reason, the amount of RANK expression in bone metastases seems to be histotype-dependent. In fact, we found the same concordance in RANK expression when we compared only primary and related bone metastasis pairs, excluding the bone metastases whose the corresponding primary tumors samples were not available.

There are no previous studies comparing RANK expression in bone metastatic tissue versus primary tumor tissue from the same patient. If we consider previous series (comparing bone metastasis with primary tumor from different patients), as for prostate cancer, our results differ from those obtained by Chen et al. (2006). In particular, their study showed a greater RANK expression in bone metastases compared with primary cancer, while our work demonstrated a very similar level of expression in primary cancer and in bone metastatic lesions. On the contrary, regarding breast cancer, our data are consistent with the observations from Bhatia et al. (2005), showing an almost complete concordance of RANK expression between primary tumors and related bone metastases.

In conclusion, on the basis of our data, RANK is expressed in a large range of primary tumors and related bone metastases. Whether this expression could represent a valid therapeutic target, not only in bone metastasis management, but also as treatment in advanced and adjuvant setting, is still an unanswered question that needs to be investigated by prospective randomized clinical trials.

\section{Literature Cited}

Bhatia P, Sanders MM, Hansen MF. 2005. Expression of receptor activator of nuclear factorkappaB is inversely correlated with metastatic phenotype in breast carcinoma. Clin Cancer Res II:162-165.

Boyce BF, Xing L. 2008. Functions of RANKL/RANK/OPG in bone modeling and remodeling. Arch Biochem Biophys 473:139-146.

Brown JM, ZhangJ, Keller ET. 2004. Opg, RANKI, and RANK in cancer metastasis: Expression and regulation. Cancer Treat Res I 18:149-172.

Chen G, Sircar K, Aprikian A, Potti A, Goltzman D, Rabbani SA. 2006. Expression of RANKL/ RANK/OPG in primary and metastatic human prostate cancer as markers of disease stage and functional regulation. Cancer 107:289-298.

Cummings SR, San Martin J, McClung MR, Siris ES, Eastell R, Reid IR, Delmas P, Zoog HB, Austin M, Wang A, Kutilek S, Adami S, Zanchetta J, Libanati C, Siddhanti S, Christiansen C. 2009. Denosumab for prevention of fractures in postmenopausal women with osteoporosis. N Engl J Med 361:756-765.

Fizazi K, Bosserman L, Gao G, Skacel T, Markus R. 2009. Denosumab treatment of prostate cancer with bone metastases and increased urine $\mathrm{N}$-telopeptide levels after therapy with intravenous bisphosphonates: Results of a randomized phase II trial. J Urol 182: 509-515; discussion 506-515.

Hsu H, Lacey DL, Dunstan CR, Solovyev I, Colombero A, Timms E, Tan HL, Elliott G, Kelley M], Sarosi I, Wang L, Xia XZ, Elliott R, Chiu L, Black T, Scully S, Capparelli C, Morony S, Shimamoto G, Bass MB, Boyle WJ. 1999. Tumor necrosis factor receptor family member RANK mediates osteoclast differentiation and activation induced by osteoprotegerin ligand. Proc Natl Acad Sci USA 96:3540-3545

Jones DH, Nakashima T, Sanchez OH, Kozieradzki I, Komarova SV, Sarosi I, Morony S, Rubin E, Sarao R, Hojilla CV, Komnenovic V, Kong YY, Schreiber M, Dixon SJ, Sims SM, Khokha R, Wada T, Penninger JM. 2006. Regulation of cancer cell migration and bone metastasis by RANKL. Nature 440:692-696.

Lau YS, Danks L, Sun SG, Fox S, Sabokbar A, Harris A, Athanasou NA. 2007. RANKLdependent and RANKL-independent mechanisms of macrophage-osteoclast differentiation in breast cancer. Breast Cancer Res Treat 105:7-16.

Mori K, Berreur M, Blanchard F, Chevalier C, Guisle-Marsollier I, Masson M, Redini F, Heymann D. 2007a. Receptor activator of nuclear factor-kappaB ligand (RANKL) directly modulates the gene expression profile of RANK-positive Saos-2 human osteosarcoma cells. Oncol Rep 18:1365-137|.

Mori K, Le Goff B, Charrier C, Battaglia S, Heymann D, Redini F. 2007b. DUI45 human prostate cancer cells express functional receptor activator of NFkappaB: New insights in the prostate cancer bone metastasis process. Bone 40:98I-990.

Nakagawa N, Kinosaki M, Yamaguchi K, Shima N, Yasuda H, Yano K, Morinaga T, Higashio K. 1998. RANK is the essential signaling receptor for osteoclast differentiation factor in osteoclastogenesis. Biochem Biophys Res Commun 253:395-400.

Sasaki A, Ishikawa K, Haraguchi N, Inoue H, Ishio T, Shibata K, Ohta M, Kitano S, Mori M. 2007. Receptor activator of nuclear factor-kappaB ligand (RANKL) expression in hepatocellular carcinoma with bone metastasis. Ann Surg Oncol 14:1|19|-1199.

Smith MR, Egerdie B, Hernandez Toriz N, Feldman R, Tammela TL, Saad F, Heracek J, Szwedowski M, Ke C, Kupic A, Leder BZ, Goessl C. 2009. Denosumab in men receiving androgen-deprivation therapy for prostate cancer. N Engl J Med 361:745-755.

Srivastava S, Matsuda M, Hou Z, Bailey JP, Kitazawa R, Herbst MP, Horseman ND. 2003. Receptor activator of NF-kappaB ligand induction via Jak2 and Stat5a in mammary epithelial cells. J Biol Chem 278:46I7I-46I78.

van Ravenswaay Claasen HH, Kluin PM, Fleuren GJ. 1992. Tumor infiltrating cells in human cancer. On the possible role of CD I6+ macrophages in antitumor cytotoxicity. Lab Invest 67:166-174.

Wittrant Y, Lamoureux F, Mori K, Riet A, Kamijo A, Heymann D, Redini F. 2006. RANKL directly induces bone morphogenetic protein-2 expression in RANK-expressing POS-1 osteosarcoma cells. Int J Oncol 28:261-269. 